(c) 2016 International Association for Ecology and Health

\title{
Editorial
}

\section{One Health: EcoHealth 2016: Welcome from the President of the International Association for Ecology and Health}

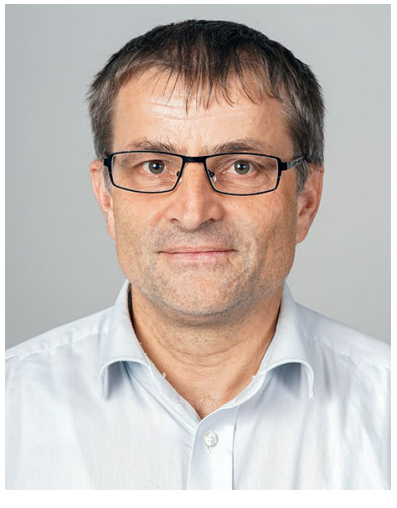

It is with great pleasure and highest esteem and appreciation for the organizing teams that the International Association for Ecology and Health welcomes you to One Health-EcoHealth 2016 in Melbourne, Australia from December 3-7. This congress brings together for the first time the global One Health and

EcoHealth communities. One Health and EcoHealth both aim for more integrated approaches to the health of humans and animals while considering the integrity of their surrounding social-ecological systems. This represents a counter current to the main stream of rather "reductionist" biomedical science, which continues to specialize and fragment. It is obvious that science progresses in ways that cannot be foreseen, in the same way that evolutionary lineages respond to a changing environment. On the other hand, we have evidence that a specialist perspective can become inadequate for solving complex problems like feeding our world safely and sustainably, responding to emerging diseases and invasive species, or addressing climate change and antimicrobial resistance.

One Health and EcoHealth are composite terms, referring to the commonality of human and animal health and the strong linkage of health with the integrity of social and ecological systems. While this seems intuitively logical, as in any other field of science, both approaches require a body of theory that underpins generic methods and approaches. Unfortunately, there remains a deficit of theory for One Health and EcoHealth. We live in a world full of "buzz words,", which are all too often hawked in a poorly reflected way causing an unnecessary lack of credibility and recognition. We very much hope that this joint conference is an opportunity to consolidate the underlying theories of One Health and EcoHealth such that they become coherent and contribute to revolutionize prevailing views on the health of humans, animals, and our planet.

From an anthropocentric perspective, animals are seen as part of the human environment. In turn, from an integrated perspective, humans and animals are part of their surrounding ecosystem. The term "health" is often applied as a metaphor to address the issue of "integrity," "stability," or "sustainability" of an ecosystem. We don't enter into the debate on the sustainability of ecosystems, but we know that polluted water and air are harmful for human and animal health. Degraded pastures cannot support livestock production, and fish contaminated with mercury are a hazard if consumed. Thus, understanding the health of humans and animals as it is intimately linked to the quality of the ecosystem and environment in which they live can provide solutions to these complex issues. This ecosystemic approach to health is, in other words, an EcoHealth approach. It follows that One Health, considered as evidence of the benefits of a closer cooperation of human and animal health, is clearly embedded within this EcoHealth framework.

The challenge is to provide the evidence of mutual or synergistic benefits of the interacting fields of human and animal health, and social and ecological sciences. In our experience, proximal linkages, for example, of pathogens transmissible between animals and humans, can be easily demonstrated. However, it becomes increasingly difficult to 
demonstrate more distal relationships in a complex animal health-ecosystem linkage at different scales. For example, we can easily demonstrate the dependence of human vitamin A status on the vitamin A content of consumed cow milk in mobile pastoralists in Chad. However, it is much more difficult to demonstrate the dependence of cow milk on the beta-carotene content of the grass the cows graze, although we know there is linkage from grass to milk to human vitamin A status. Understanding such linkages are necessary requirements for both One Health and EcoHealth, but they are not, on their own, sufficient. We consider it a sufficient requirement for a One Health or an EcoHealth approach only when we can demonstrate an added value or synergistic benefit of such inter- and transdisciplinary approaches, through engaging academic and non-academic, local knowledge. One Health and EcoHealth become credible and recognized when they can demonstrate that humans and animals become healthier if doctors and veterinarians work together rather than in separation. Furthermore, as humans and animals become healthier, ecosystem services are sustained and/or financial resources are saved when other fields of science work together with health sciences. Only at this point do we establish the requirements and expectations for a genuine One Health/EcoHealth approach. This is the ideal we aspire to, although achieving this level is difficult and good and convincing examples are still rare. We continue to expand the number of examples of the direct benefits from these systemic approaches to health, including through the contributions presented in this special conference issue of the EcoHealth journal. In this way, One Health and EcoHealth will no longer be simply "buzz words" because they will be recognized by governments, authorities and communities, as well as the private sector, and will contribute to solve the enormous social and environmental challenges already present and those yet to come.

We look forward to meeting you all in December 2016.

Welcome to Melbourne!

Jakob Zinsstag, President, International Association for Ecology and Health

Jakob Zinsstag

Human and Animal Health Unit, Department of Epidemiology and Public Health, Swiss Tropical and Public Health Institute, PO Box, 4002 Basel, Switzerland e-mail: jakob.zinsstag@unibas.ch

Published online: October 3, 2016 